\title{
The association of adiponectin with risk of pre-diabetes and diabetes in different subgroups: cluster analysis of a general population in south China
}

Xun Gong 1,*, Lili You ${ }^{1, *}$, Feng Li ${ }^{1}$, Qingyu Chen², Chaogang Chen ${ }^{3}$, Xiaoyun Zhang ${ }^{1}$, Xiuwei Zhang ${ }^{4}$, Wenting Xuan ${ }^{4}$, Kan Sun ${ }^{1}$, Guojuan Lao ${ }^{1}$, Chuan Wang ${ }^{1}$, Yan Li ${ }^{1}$, Mingtong $\mathrm{Xu}^{1}$, Meng Ren ${ }^{1, \dagger}$ and Li Yan ${ }^{1, \dagger}$

1'Department of Endocrinology, Sun Yat-sen Memorial Hospital, Sun Yat-sen University, Guangzhou, People's Republic of China 2Department of Medical Examination Center, Sun Yat-sen Memorial Hospital, Sun Yat-sen University, Guangzhou, People's Republic of China ${ }^{3}$ Department of Clinical Nutrition, Sun Yat-sen Memorial Hospital, Sun Yat-sen University, Guangzhou, People's Republic of China

${ }^{4}$ Department of Endocrinology, Dongguan People's Hospital, Dongguan, People's Republic of China

Correspondence should be addressed to M Ren or L Yan: renmeng80@139.com or yanli@mail.sysu.edu.cn

*(X Gong and L You contributed equally to this work and should be regarded as co-first authors)

${ }^{\dagger}$ (M Ren and L Yan contributed equally to this work as co-corresponding authors)

\begin{abstract}
Objective: Adiponectin is an adipocyte-derived hormone with an important role in glucose metabolism. The present study explored the effect of adiponectin in diverse population groups on pre-diabetes and newly diagnosed diabetes.

Methods: A total of 3300 individuals were enrolled and their data were collected in the analyses dataset from December 2018 to October 2019. Cluster analysis was conducted based on age, BMI, waistline, body fat, systolic blood pressure, triglycerides, and glycosylated hemoglobin $1 c$. Cluster analysis divided the participants into four groups: a young-healthy group, an elderly-hypertension group, a high glucose-lipid group, and an obese group. Odds ratio (OR) and $95 \% \mathrm{Cls}$ were calculated using multivariate logistic regression analysis.

Results: Compared with the first quartile of adiponectin, the risk of pre-diabetes of fourth quartile was decreased $61 \%(\mathrm{aOR}=0.39,95 \% \mathrm{Cl}(0.20-0.73)$ ) in the young-healthy group; and the risk of diabetes of fourth quartile was decreased $85 \%(\mathrm{aOR}=0.15$, $95 \% \mathrm{Cl}(0.02-0.67))$ in the obese group. There were no significant correlations between the adiponectin level and diabetes/pre-diabetes in the other two groups. Additionally, receiver operating characteristic curve analysis indicated that adiponectin could significantly improve the diagnosis based on models in the young-healthy group (from 0.640 to 0.675 ) and the obese group (from 0.714 to 0.761 ).

Conclusions: Increased adiponectin levels were associated with decreased risk of prediabetes in the young-healthy population, and with a decreased the risk of diabetes in the obese population. An increased adiponectin level is an independent protective factor for pre-diabetes and diabetes in a specific population in south China.
\end{abstract}

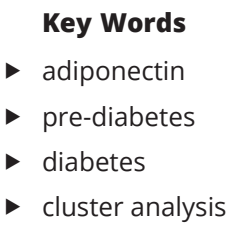

Endocrine Connections (2021) 10, 1410-1419 https://ec.bioscientifica.com https://doi.org/10.1530/EC-21-0235

C) 2021 The authors Published by Bioscientifica Ltd

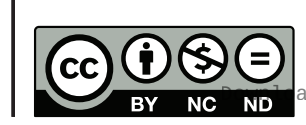

This work is licensed under a Creative Commons Attribution-NonCommercial-NoDerivatives 4.0 elnternationab ticense.ifica.com at 04/26/2023 02:20:22PM 


\section{Introduction}

Diabetes mellitus is a global public health issue that causes huge economic and medical burdens worldwide. The prevalence of diabetes has increased over the past few decades, especially in Asian population (1). A national cross-sectional study, which included 75,880 Chinese adults showed that the prevalence of total diabetes and prediabetes, according to the American Diabetes Association (ADA) criteria, reached 12.8 and $35.2 \%$, respectively (2). Moreover, $70 \%$ of patients with pre-diabetes will eventually develop full diabetes, according to an ADA expert panel (3).

Adiponectin, one of the most abundant peptides secreted specifically by adipocytes, has been shown to improve insulin resistance and inflammatory status (4). Adiponectin reduction plays a central role in obesityrelated diseases, including insulin resistance, type 2 diabetes mellitus (T2DM), lipid homeostasis, and cardiovascular disease $(5,6,7)$. Accumulating evidence shows that higher adiponectin levels are associated with lower risk of T2DM (8). A meta-analysis including 15 case-control studies indicated that the circulating level of adiponectin might decrease before the onset of diabetes (9). Therefore, it is important for investigators to have a thorough understanding of adiponectin and its effect on pre-diabetes and diabetes.

However, evidence for the effect of adiponectin on pre-diabetes or diabetes in diverse populations is sparse. A cohort study conducted in elderly patients in Korea suggested that obesity, insulin resistance, and aging are closely related (10). That study found that plasma adiponectin levels were lower in elderly pre-diabetes individuals than those with normal glucose tolerance. A 3 -year prospective study enrolled 175 overweight and obese subjects found that the plasma adiponectin concentration was a sensitive predictor of impaired fasting glucose (IFG) (11). However, the number of subjects was relatively small. Another study included 91 middle-aged Asian Indian subjects observed that a low adiponectin concentration was a strong predictor of future development of diabetes in subjects with impaired glucose tolerance (IGT) (12). However, their conclusions might not be applicable to other populations.

Cluster analysis refers to a group of multivariate mathematical algorithms, which has the advantage of producing a more adjustable classification and is widely used in clinical research statistical analysis $(13,14)$. The present study enrolled 3300 individuals with the aim of exploring the effect of adiponectin in different characteristic groups using cluster analysis on pre-diabetes and newly diagnosed diabetes. Further analysis of subgroups might provide new evidence linking adiponectin to diabetes development.

\section{Subjects and methods}

\section{Study populations}

The study population was collected by a cross-sectional study in Guangzhou and Dongguan, China, from December 2018 to October 2019. The enrolled study population in Guangzhou came from the Sun Yat-sen Memorial Hospital of Physical Examination Center. Meanwhile, the population recruited in Dongguan mainly came from communities (Dalingshan community, Zhangmu community, Daojiao community, Qiaotou community, Songshan lake community, Qingxi community, Zhang'an community, and Meinian Physical Examination Center). Subjects that met the inclusion criteria were asked to complete a questionnaire, and were subjected to physical examination, laboratory tests, and serum adiponectin measurement. Inclusion criteria: (i) 18-70 years old; (ii) Chinese Han population; and (iii) residents who lived in those regions for $\geq 3$ years. Exclusion criteria: (i) Pregnant women; (ii) those who suffer from severe diseases, such as hepatic cirrhosis, chronic renal failure, or evident cardiac insufficiency; (iii) those with a history of infectious disease; (iv) those with a history of malignant tumors; (v) those with diagnosed as hypertension, hyperlipidemia, or cardiovascular and cerebrovascular diseases; (vi) those with other endocrine diseases; (vii) those with mental illness; and (viii) those who have used drugs, dietary supplement, functional foods for a long time ( $\geq 3$ times/week for more than 3 months).

During the recruitment phase, 3866 individuals signed the informed consent form and were enrolled for this study. Subjects who failed to provide blood glucose information ( $n=171)$ and with a history of diabetes $(n=13)$ were excluded. In addition, individuals who failed to provide information (age, $n=10$; fasting blood glucose (FBG), $n=2$; BMI, $n=43$; systolic blood pressure (SBP), $n=105$; triglycerides (TG), $n=14$; waistline, $n=195$, or body fat, $n=218$ ) were excluded from this analysis dataset. A total of 3300 subjects were included in the final data analyses. Written informed consent was obtained from each participant before data collection.

\section{Measurements}

All participants completed anthropometrical measurement with the assistance of trained staff using 
standard protocols. The height and weight are recorded separately, and the units were accurate to $0.1 \mathrm{~cm}$ and 0.1 $\mathrm{kg}$, respectively. Participants were required to remove their shoes and wear light indoor clothing during the measurement. BMI was calculated as weight in kilograms divided by height in meters squared $\left(\mathrm{kg} / \mathrm{m}^{2}\right)$. Waist circumference (WC) was measured at the umbilicus and the hipline was measured at the widest level over the great trochanters with measurement accuracy of $0.1 \mathrm{~cm}$. Blood pressure measurements were obtained using an automated electronic device (OMRON, Omron Company, Shanghai, China) after the subjects had sat for $5 \mathrm{~min}$.

Venous blood samples were collected and stored at $-80^{\circ} \mathrm{C}$ for laboratory tests after an overnight fasting of at least $10 \mathrm{~h}$. Measurement of fasting plasma glucose (FPG), TG, total cholesterol (TC), LDL-C, and HDL-C were performed using an autoanalyser (Beckman CX-7 Biochemical Autoanalyser, Brea, CA, USA). HemoglobinA1c (HbA1c) was assessed by HPLC (BioRad). Adiponectin was detected by latex-enhanced immunoturbidimetry (catalog number: 20182400947) using automatic biochemical analyzer (Mindray, 20152401201). The intraassay coefficients of variation (CV) were less than $10.0 \%$, and the interassay CVs were less than $15.0 \%$.

\section{Definitions of pre-diabetes and diabetes}

According to the ADA diagnostic criteria (15), diabetes is defined as FPG $\geq 7.0 \mathrm{mmol} / \mathrm{L}$ or $\mathrm{HbA} 1 \mathrm{C} \geq 6.5 \%$. Pre-diabetes including IFG and/or IGT. IFG defined as FPG value $\geq 5.6$ $\mathrm{mmol} / \mathrm{L}$ but $<6.9 \mathrm{mmol} / \mathrm{L}$ and $\mathrm{HbA} 1 \mathrm{C}>5.7 \mathrm{mmol} / \mathrm{L}$ but $<6.4 \mathrm{mmol} / \mathrm{L}$. IGT defined as FPG value $<7.0 \mathrm{mmol} / \mathrm{L}$ and $\mathrm{HbA} 1 \mathrm{C}>5.7 \mathrm{mmol} / \mathrm{L}$ but $<6.4 \mathrm{mmol} / \mathrm{L}$.

\section{Cluster analysis}

Model variables were selected based on the representativeness of disease features and their clinical relevance. Age (years), BMI $\left(\mathrm{kg} / \mathrm{m}^{2}\right)$, waistline $(\mathrm{cm})$, body fat $(\%)$, SBP $(\mathrm{mmHg}), \mathrm{TG}(\mathrm{mmol} / \mathrm{L})$, and glycosylated hemoglobin 1c (HbA1c) (\%) were chosen for cluster analysis. Cluster analysis was performed on values centered to a mean value of 0 and a S.D. of 1 . Two-Step clustering, in which the first step estimates the optimal number of clusters on the basis of the silhouette width and the second step performs hierarchical clustering, was carried out in R-Studio version 3.6.1 for 2-15 clusters. K-means clustering was performed with a $\mathrm{k}$ value of 4 using the kmeansruns function (runs $=100$ ) in the fpc packages.

\section{Statistical analysis}

Continuous variables with a normal distribution are presented as mean \pm S.D. Differences between groups were tested using one-way ANOVA, and post hoc comparisons were performed using Bonferroni correction. Continuous variables with a non-normal distribution are presented as medians (interquartile ranges) and difference among groups were assessed using a Kruskal-Wallis test. Categorical variables are expressed as numbers (proportions). Comparisons between categorical variables were performed using the $\chi^{2}$ test. Unadjusted and multivariate-adjusted logistic regression analysis was used to assess the factors influencing the increased prevalence of pre-diabetes and diabetes. Odds ratios (ORs) and their corresponding 95\% CIs were calculated using logistic regression models. Nomograms were established based on the results of multivariable analysis. Receiver operating characteristic (ROC) curves were plotted in order to investigate the associations of adiponectin with prediabetes in cluster 1 and diabetes in cluster 4 . All statistical analyses were performed using R-Studio version 3.6.1. Statistical tests were two-sided, and a $P$ value $<0.05$ was considered statistically significant.

\section{Results}

\section{Cluster analysis}

A total of 3300 subjects were included in the analysis dataset. Age (years), BMI $\left(\mathrm{kg} / \mathrm{m}^{2}\right)$, waistline $(\mathrm{cm})$, body fat (\%), SBP (mmHg), TG (mmol/L), and HbA1c (\%) were chosen for cluster analysis. The subjects were classified into four novel subgroups using cluster analysis. Cluster 1, including 1298 (39.3\%) of 3300 clustered subjects, was termed the young-healthy population, which was characterized by young age, relatively low obesity-related indicators (BMI, waistline, and body fat), and a normal range of SBP, HbA1c, and TG levels. Cluster 2, including 930 $(28.2 \%)$ subjects and was termed the elderly hypertension population, which was characterized by older age, high SBP levels, relatively low obesity-related indicators (BMI, waistline, and body fat), normal glucose (HbA1c), and lipid (TG) levels. Cluster 3, termed the high glucose-lipid population, included 49 (1.5\%) subjects, was characterized by high levels of HbA1c and TG levels, older age, and

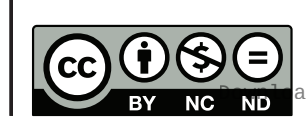

This work is licensed under a Creative Commons Attribution-NonCommercial-NoDerivatives 4.0 Internationab ficense.ifica.com at 04/26/2023 02:20:22PM 
relatively high levels of obesity-related indicators and SBP. Cluster 4, including 1023 (31.0\%) subjects, was termed the obese population and was characterized by high BMI, waistline, and body fat, and relatively high glucose-lipid and SBP levels. Distributions of age, HbA1c, BMI, waistline, body fat, SBP, and TG in the analysis dataset for clusters are shown in Fig. 1. The basic clinical characteristics according to the clusters are presented in Table 1.

\section{Multivariate regression analysis}

Logistic regression analysis was used to explore the relationship between adiponectin and pre-diabetes or diabetes in the different populations (Table 2). In the total dataset, compared with subjects in quantile 1 of the adiponectin level, the unadjusted model showed that subjects in quantiles $2-4$ had a significant correlation with decreased odds of pre-diabetes and diabetes. In addition, adiponectin was identified as an independent protective factor of pre-diabetes and diabetes after adjustment by confounding factors, including sex, age, BMI, waistline, body fat, and SBP. However, the independent protective effect of adiponectin was weakened when further adjusted by TG and LDL-C (the $P$-values were 0.014 and 0.038 , respectively, for pre-diabetes and diabetes, respectively).

We further investigated the relationship between adiponectin and pre-diabetes and diabetes in the different clusters (Table 2). As the quantile of adiponectin increased, the odds of pre-diabetes decreased in cluster 1 after adjusted by models $1-5$. In model 5 , the ORs of the decrease in prediabetes with increasing adiponectin quantile were 1.00 (reference), 0.82 (95\% CI 0.50-1.35; quantile 2), 0.59 (95\% CI 0.34-1.02; quantile 3), and 0.39 (95\% CI 0.20-0.73; quantile 4). Similar trend was detected in cluster 4, the ORs of decreased diabetes with increasing adiponectin quantile were 1.00 (reference), 0.88 (95\% CI 0.40-1.90; quantile 2), 0.44 (95\% CI 0.14-1.20; quantile 3), and 0.15 (95\% CI 0.02-0.67; quantile 3). The results showed that adiponectin was significantly associated with diabetes but not for pre-diabetes in cluster 4 . However, there were no correlation between adiponectin and pre-diabetes or diabetes in clusters $2-3$ in our study. The nomogram models are shown in Figs 2 and 3.

\section{Nomograms and ROC curves}

The associations of adiponectin with pre-diabetes in cluster 1 and diabetes in cluster 4 were further explored using nomogram models and ROC curves. As shown in Figs 2 and 3, significant influencing factors in the multivariate logistic model (age, sex, waistline, BMI, SBP, body fat, TG, and adiponectin) were included in a nomogram model to evaluate the risk of pre-diabetes and diabetes, respectively. By taking a 30-year-old male as an example, drawing an upward vertical line from the age variable axis to the Point bar gets a point of 10 . Male gets a point of 30 . If the adiponectin of this individual is $4 \mathrm{mg} / \mathrm{L}$, the point corresponding to the adiponectin variable is 78 . If the waistline is $85 \mathrm{~cm}$, the corresponding point is 40 . If the BMI is $24 \mathrm{~kg} / \mathrm{m}^{2}$, the corresponding point is 35 . If the SBP is $120 \mathrm{mmHg}$, the corresponding point is 14 . If the body fat is $20 \%$, the corresponding point is 43 . If the TG is $3.6 \mathrm{mmol} / \mathrm{L}$, the corresponding point is 30 . The total points equal to $280(10+30+78+40+35+14+43+30)$. Thus, the risk of pre-diabetes is approximately $20 \%$ when we draw a straight line from the total point axis to the risk of
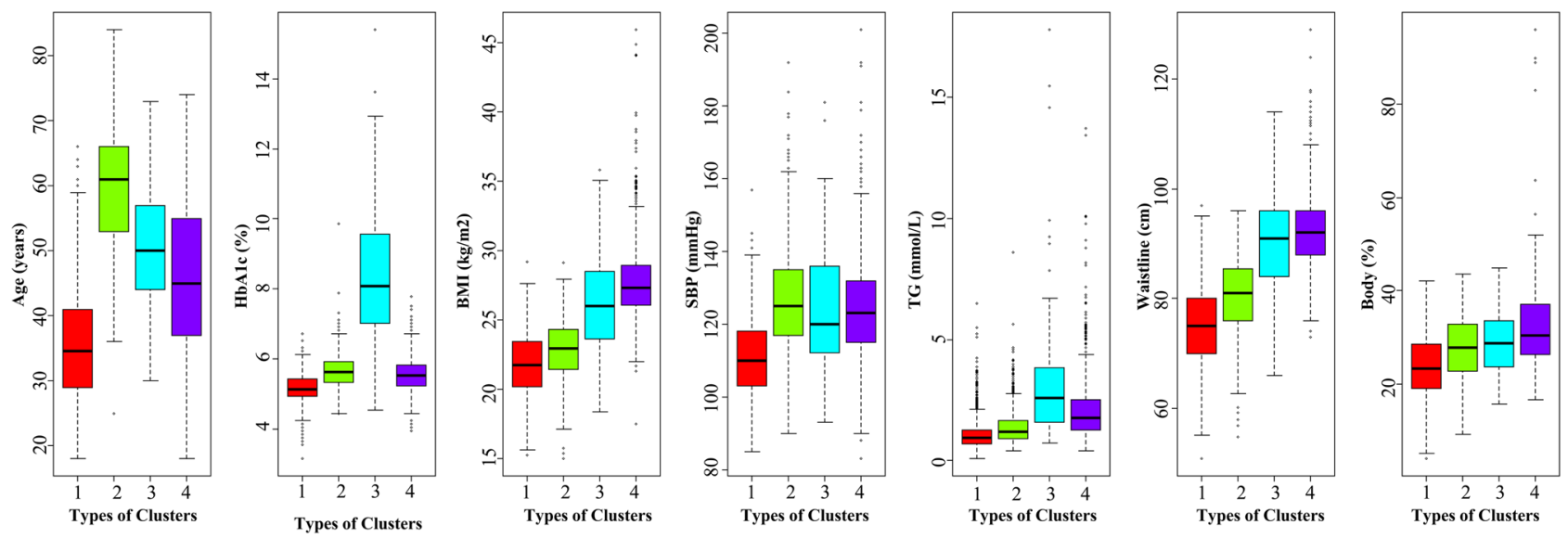

Figure 1

Distribution of the analysis dataset in the types of clusters. Red, data for cluster 1; green, data for cluster 2; blue, data for cluster 3; and purple, data for cluster 4.

https://ec.bioscientifica.com https://doi.org/10.1530/EC-21-0235
(C) 2021 The authors Published by Bioscientifica Ltd

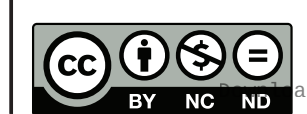

This work is licensed under a Creative Commons Attribution-NonCommercial-NoDerivatives 4.0 International dicense ifica. com at 04/26/2023 02:20:22PM 
Table 1 Clinical and anthropometric characteristics of different clusters.

\begin{tabular}{l}
\hline Variables \\
\hline Gender \\
$\quad$ Male \\
$\quad$ Female \\
Age (years) \\
Height $(\mathrm{cm})$ \\
Weight $(\mathrm{kg})$ \\
BMI $\left(\mathrm{kg} / \mathrm{m}^{2}\right)$ \\
Waistline (cm) \\
Hipline (cm) \\
WHR \\
Body fat (\%) \\
SBP (mmHg) \\
DBP (mmHg) \\
TC (mmol/L) \\
TG (mmol/L) \\
LDL-C (mmol/L) \\
HDL-C (mmol/L) \\
Adiponectin (mg/L) \\
FBG (mmol/L) \\
HbA1c (\%)
\end{tabular}

\begin{tabular}{c}
\hline \\
\hline Cluster 1 \\
\hline \\
$521(33.2)$ \\
$777(44.9)$ \\
$35 \pm 8.8$ \\
$162.3 \pm 8.2$ \\
$57.6 \pm 9.0$ \\
$21.8 \pm 2.3$ \\
$74.7 \pm 7.4$ \\
$91.2 \pm 5.9$ \\
$0.82 \pm 0.06$ \\
$23.6 \pm 6.2$ \\
$111 \pm 10.7$ \\
$69 \pm 8.0$ \\
$4.82 \pm 0.91$ \\
$1.10 \pm 0.62$ \\
$2.79 \pm 0.73$ \\
$1.51 \pm 0.39$ \\
$4.55 \pm 1.90$ \\
$4.76 \pm 0.48$ \\
$5.14 \pm 0.38$ \\
\hline
\end{tabular}

\begin{tabular}{c} 
Types \\
Cluster 2 \\
\hline \\
$431(27.4)$ \\
$499(28.9)$ \\
$59 \pm 8.2^{\mathrm{a}}$ \\
$159.5 \pm 8.7^{\mathrm{a}}$ \\
$58.1 \pm 8.5$ \\
$22.8 \pm 2.1^{\mathrm{a}}$ \\
$80.6 \pm 6.7^{\mathrm{a}}$ \\
$92.6 \pm 5.2^{\mathrm{a}}$ \\
$0.87 \pm 0.06^{\mathrm{a}}$ \\
$27.6 \pm 6.2^{\mathrm{a}}$ \\
$126 \pm 14.2^{\mathrm{a}}$ \\
$76 \pm 9.3^{\mathrm{a}}$ \\
$5.45 \pm 1.02^{\mathrm{a}}$ \\
$1.38 \pm 0.74^{\mathrm{a}}$ \\
$3.25 \pm 0.91^{\mathrm{a}}$ \\
$1.57 \pm 0.62$ \\
$5.32 \pm 2.75^{\mathrm{a}}$ \\
$5.12 \pm 0.64^{\mathrm{a}}$ \\
$5.63 \pm 0.46^{\mathrm{a}}$
\end{tabular}

\begin{tabular}{c} 
of clusters \\
\hline Cluster 3 \\
\hline $29(1.9)$ \\
$20(1.2)$ \\
$51 \pm 10.2^{\mathrm{ab}}$ \\
$163.1 \pm 7.9^{\mathrm{b}}$ \\
$70.9 \pm 13.8^{\mathrm{ab}}$ \\
$26.5 \pm 3.9^{\mathrm{ab}}$ \\
$90.5 \pm 10.9^{\mathrm{ab}}$ \\
$98.3 \pm 6.9^{\mathrm{ab}}$ \\
$0.92 \pm 0.07^{\mathrm{ab}}$ \\
$29.0 \pm 6.4^{\mathrm{a}}$ \\
$126 \pm 20.1^{\mathrm{a}}$ \\
$77 \pm 10.1^{\mathrm{a}}$ \\
$5.86 \pm 1.46^{\mathrm{ab}}$ \\
$3.92 \pm 3.85^{\mathrm{ab}}$ \\
$3.21 \pm 1.19^{\mathrm{a}}$ \\
$1.35 \pm 0.60^{\mathrm{b}}$ \\
$2.92 \pm 1.66^{\mathrm{ab}}$ \\
$10.22 \pm 3.19^{\mathrm{ab}}$ \\
$8.38 \pm 2.10^{\mathrm{ab}}$
\end{tabular}

\begin{tabular}{c} 
\\
\hline Cluster 4 \\
\hline $590(37.6)$ \\
$433(25.0)$ \\
$46 \pm 12.3^{\mathrm{abc}}$ \\
$163.0 \pm 9.0^{\mathrm{b}}$ \\
$73.8 \pm 10.3^{\mathrm{ab}}$ \\
$27.7 \pm 2.7^{\mathrm{abc}}$ \\
$92.3 \pm 7.0^{\mathrm{ab}}$ \\
$101.2 \pm 5.8^{\mathrm{abc}}$ \\
$0.91 \pm 0.06^{\mathrm{ab}}$ \\
$31.9 \pm 7.4^{\mathrm{abc}}$ \\
$125 \pm 14.4^{\mathrm{a}}$ \\
$77 \pm 10.3^{\mathrm{ab}}$ \\
$5.31 \pm 0.12^{\mathrm{ac}}$ \\
$1.34 \pm 2.09^{\mathrm{ac}}$ \\
$3.20 \pm 0.94^{\mathrm{a}}$ \\
$1.40 \pm 0.64^{\mathrm{ab}}$ \\
$3.43 \pm 1.66^{\mathrm{ab}}$ \\
$5.14 \pm 0.69^{\mathrm{ac}}$ \\
$5.50 \pm 0.48^{\mathrm{abc}}$
\end{tabular}

\begin{tabular}{|c|c|}
\hline$F / \chi^{2}$ & $P$ \\
\hline 73.814 & $<0.001$ \\
\hline 225.6 & $<0.001$ \\
\hline 8.203 & 0.004 \\
\hline 1799.00 & $<0.001$ \\
\hline 3651.00 & $<0.001$ \\
\hline 3553.00 & $<0.001$ \\
\hline 1824.00 & $<0.001$ \\
\hline 1403.00 & $<0.001$ \\
\hline 879.90 & $<0.001$ \\
\hline 489.30 & $<0.001$ \\
\hline 396.50 & $<0.001$ \\
\hline 108.90 & $<0.001$ \\
\hline 549.7 & $<0.001$ \\
\hline 103.20 & $<0.001$ \\
\hline 27.69 & $<0.001$ \\
\hline 201.50 & $<0.001$ \\
\hline 138.20 & $<0.001$ \\
\hline 209.90 & $<0.001$ \\
\hline
\end{tabular}

Data were expressed as mean \pm S.D., all of the $P$ value were calculated by post hoc comparisons using Bonferroni correction. Bold indicates statistical significance.

${ }^{a} P<0.05$ compared with subjects in cluster $1 ;{ }^{b} P<0.05$ compared with subjects in cluster $2 ;{ }^{c} P<0.05$ compared with subjects in cluster 3 .

pre-diabetes axis (Fig. 2). Moreover, our study also investigated the association of adiponectin with prediabetes in cluster 1 and diabetes in cluster 4 using ROC curves (Fig. 4). As shown in Fig. 4A, compared with the combined mode (black), which included age, BMI, waistline, body fat, and SBP, the area under the ROC curve (AUC) valve increased from 0.640 to 0.682 when the indicators (TG, LDL-C, and adiponectin) were added to the combined mode. Similarly, the AUC valve increased from 0.724 to 0.779 when the combined mode added TG, LDL-C, and adiponectin, as shown in Fig. 4B.

\section{Discussion}

The present study enrolled 3300 individuals and explored the effect of adiponectin on pre-diabetes and newly diagnosed diabetes using cluster analysis. Several interesting and important points were revealed by our study. Adiponectin was identified as an independent protective factor for pre-diabetes in young-healthy adults, as well as for diabetes in obesity adults. However, adiponectin was not considered an independent factor for pre-diabetes or diabetes in the elderly hypertension and high glucose-lipid clusters.

Similar to previous studies, our results showed that adiponectin is correlated negatively with pre-diabetes and newly diagnosed diabetes in the total study population. These connections were weakened after additional adjustment by TG and LDL-C. It is widely recognized that hypertriglyceridemia is an important feature of lipid metabolism abnormality in patients with T2DM. Snijder's study suggested that TG substantially reduce the associations between adiponectin and the incidence of T2DM (16). Adiponectin was reported to be involved in the regulation of lipid and glucose metabolism, and could promote triglyceride-rich lipoprotein catabolism, which in return, leads to lower TG levels (17).

In the present study, increased adiponectin level had an independent protect effect on pre-diabetes in younghealthy adults. One study that recruited 542 Chinese young people demonstrated that adiponectin level was associated with isolated IGT rather than isolated IFG (18). Their study indicated that alterations in adipokine concentrations had already been detected in IGT states, which might reflect adipose tissue dysfunction as an early event in the development of T2DM. A biracial cohort study showed a decreased adiponectin level is a powerful risk marker for the incidence of pre-diabetes in healthy White and Black adults with a parental history of T2DM (19). However, the participants were the offspring of parents with T2DM and thus the results might not be applicable to individuals that without a family history of diabetes or the general population.

This work is licensed under a Creative Commons Attribution-NonCommercial-NoDerivatives 4.0 internationad bicense.ifica.com at 04/26/2023 02:20:22PM 


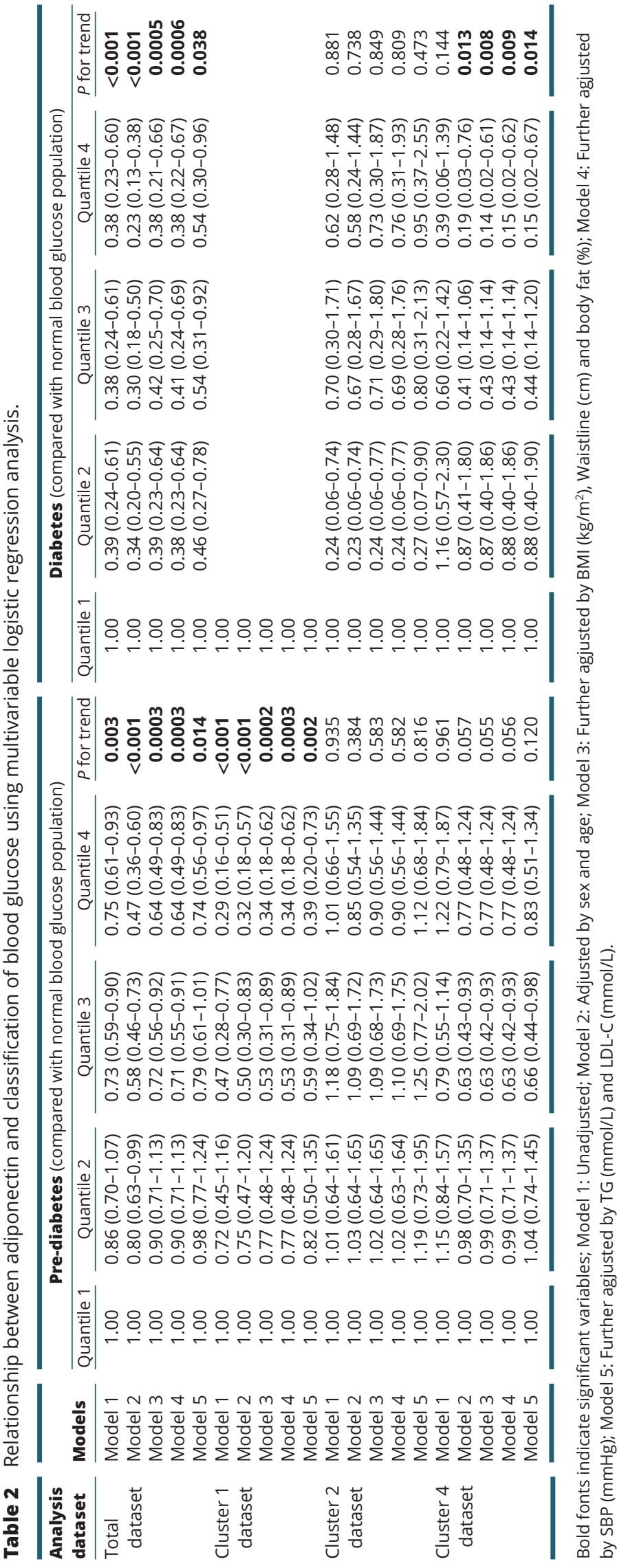

https://ec.bioscientifica.com https://doi.org/10.1530/EC-21-0235 (c) 2021 The authors Published by Bioscientifica Ltd
We found that increased adiponectin level had an independent protective effect on diabetes in obese people. Obesity-associated diabetes has caused a healthcare crisis worldwide. A 5-year prospective study of nondiabetic Japanese Americans found that higher concentration of adiponectin could reduce the accumulation of intraabdominal fat (20). Another study that enrolled 85 healthy participants and 38 newly diagnosed diabetes patients (19 with a normal BMI and 17 who were obese) indicated that the adiponectin level in newly diagnosed obese patients with T2DM was lower than those who had normal BMI after adjusting by BMI and WC (21). The study presumed that patients with T2DM who are obese exhibit more severe insulin resistance status than those with normal BMI. A previous study also stated that adiponectin replacement therapy has a preventive effect for patients with T2DM by decreasing insulin resistance (22). However, Mather's study enrolled participants receiving treatment, and it came to a different conclusion that baseline adiponectin levels were strongly and inversely related to progression to diabetes independent of obesity, which demonstrated the separate associations of weight and adiponectin with progression to diabetes (23).

There were no correlations between adiponectin and pre-diabetes or diabetes in clusters 2 and 3 in our study. Our results showed that the adiponectin levels in cluster 2 were significantly higher than those in cluster 1 , and the adiponectin levels of cluster 3 were significant lower than those in cluster 1 . However, the adiponectin level was not an independent risk factor of diabetes or pre-diabetes in cluster 2 or cluster 3 . It was observed that the adiponectin concentration is higher in older adults than young adults. One study regarded adiponectin resistance as an explanation for the higher adiponectin level with age (24). Previous research found that plasma adiponectin levels were lower in elderly subjects with pre-diabetes than in subjects with normal glucose tolerance (10). They believed that a decline in plasma adiponectin levels is accompanied by an increase in insulin resistance. A 5.5-year follow-up study including 629 Caucasian women found that a low concentration of serum adiponectin was an independent predictive factor for T2DM (25). A Portuguese study demonstrated that the adiponectin levels in elderly T2DM patients were closely linked to obesity and the duration of the disease. Plasma adiponectin levels were significant lower in elderly ( $\geq 65$ years old) T2DM subjects compared with normal subjects (26). However, no similar result was found after statistical adjustment for length of disease and BMI. In our study, cluster 3 was characterized by high levels

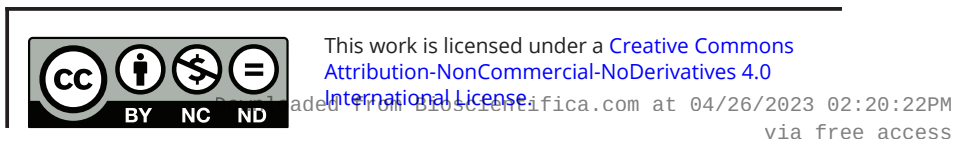


Points

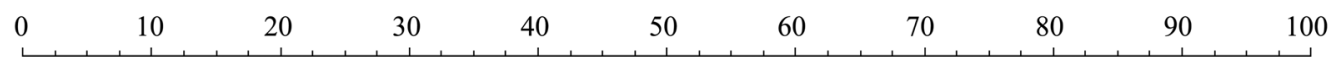

Age (years)

$152025303540 \quad 455055606570$

Sex $(1=$ Male; $2=$ Female $)$

2

Waistline (cm)

BMI $(\mathrm{kg} / \mathrm{m} 2)$

SBP (mmHg)

Bodyfat $(\%)$

\begin{tabular}{lllllllllll}
\hline 50 & 55 & 60 & 65 & 70 & 75 & 80 & 85 & 90 & 95 & 100
\end{tabular}

$\mathrm{TG}(\mathrm{mmol} / \mathrm{L})$

\begin{tabular}{|c|c|c|c|c|c|c|c|}
\hline 29 & & \multicolumn{2}{|l|}{27} & 25 & \multicolumn{2}{|c|}{24} & 22 \\
\hline
\end{tabular}

Adiponectin

$160 \quad 140 \quad 120 \quad 100 \quad 90 \quad 80$

Total Points

\begin{tabular}{llllllllll}
\hline 0 & 5 & 10 & 15 & 20 & 25 & 30 & 35 & 40 & 45
\end{tabular}

\begin{tabular}{lllllllllllllll}
\hline 0 & 0.5 & 1 & 1.5 & 2 & 2.5 & 3 & 3.5 & 4 & 4.5 & 5 & 5.5
\end{tabular}

Pre-diabetes rate

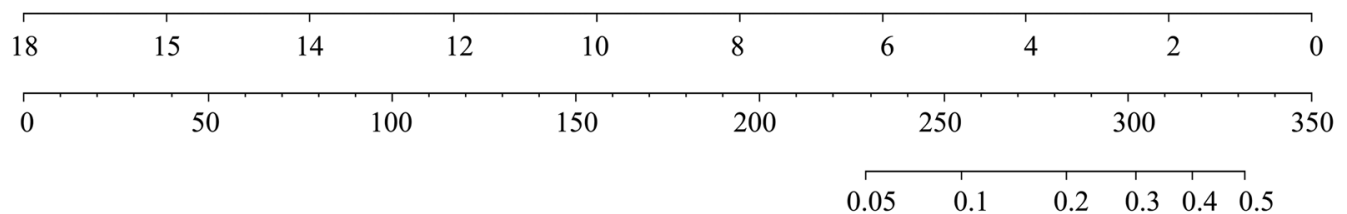

Figure 2

Factors influencing pre-diabetes in the multivariate logistic analysis in the nomogram model. According to the clinical and anthropometric characteristics of the subjects, the total points were counted. The pre-diabetes rate was matched with total points.

of $\mathrm{HbA1c}$ and TG, relatively older age, and increased levels of obesity-related indicators and SBP. HbA1c and TG levels are the two main biomarkers for metabolic syndrome. In addition, diabetes is strongly related to high glucose level
(FBG, post-prandial blood glucose (PBG), IFG, and IGT) (27). Therefore, increasing adiponectin might not be an independent protective factor of pre-diabetes or diabetes in cluster 3 .

Points

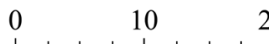

$20 \quad 30$

40

50

$\begin{array}{r}60, \quad 70, \quad 80, \quad 90 \\ \hline\end{array}$

Age (years)

Sex (1=Male; $2=$ Female $)$

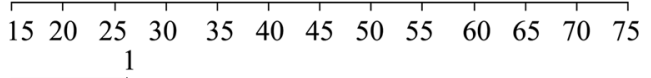

Waistline $(\mathrm{cm})$

70115

BMI $(\mathrm{kg} / \mathrm{m} 2)$

$\begin{array}{lllllllll}16 & 20 & 24 & 28 & 32 & 36 & 40 & 44\end{array}$

SBP (mmHg)

$80-180$

Bodyfat (\%)

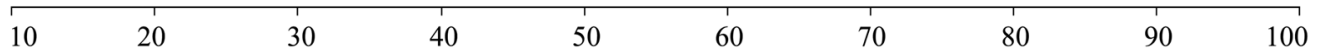

TG (mmol/L)

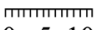

Adiponectin

\begin{tabular}{lllllllllllll}
\hline 12 & 11 & 10 & 9 & 8 & 7 & 6 & 5 & 4 & 3 & 2 & 1
\end{tabular}

Total Points

\begin{tabular}{lllrrrrrrrrrrrrr}
\hline 0 & 20 & 40 & 60 & 80 & 100 & 120 & 140 & 160 & 180 & 200 & 220 \\
& & & 0.001 & & 0.01 & 0.05 & 0.1 & 0.2 & 0.3 & 0.40 .5 & 0.6 & 0.7 & 0.8 & 0.9 & 0.95
\end{tabular}

Diabetes rate

Figure 3

Factors influencing diabetes in the multivariate logistic analysis in the nomogram model. According to the clinical and anthropometric characteristics of the subjects, the total points were counted. The diabetes rate was matched with total points. 


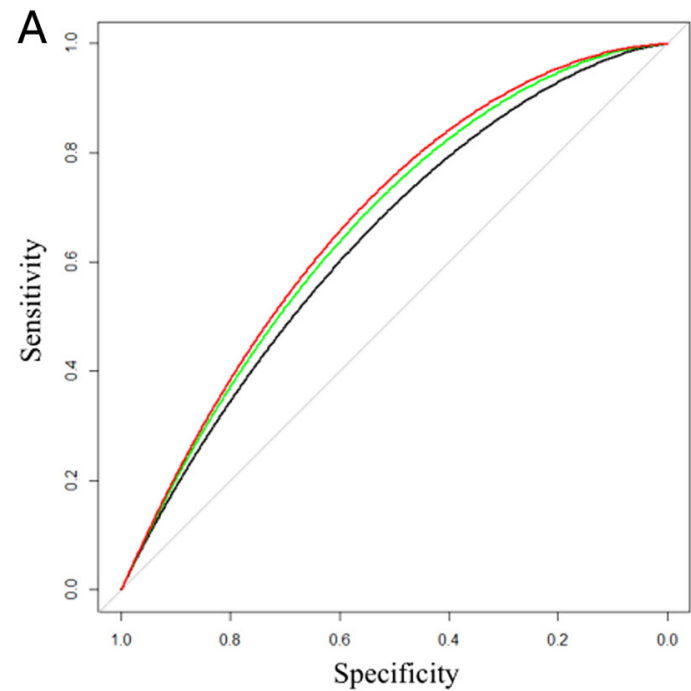

ROC Curve (Area)

Combine $(0.640)$

- Combine+TG+LDLC (0.665)

Combine+TG+LDLC+Adiponectin (0.682)

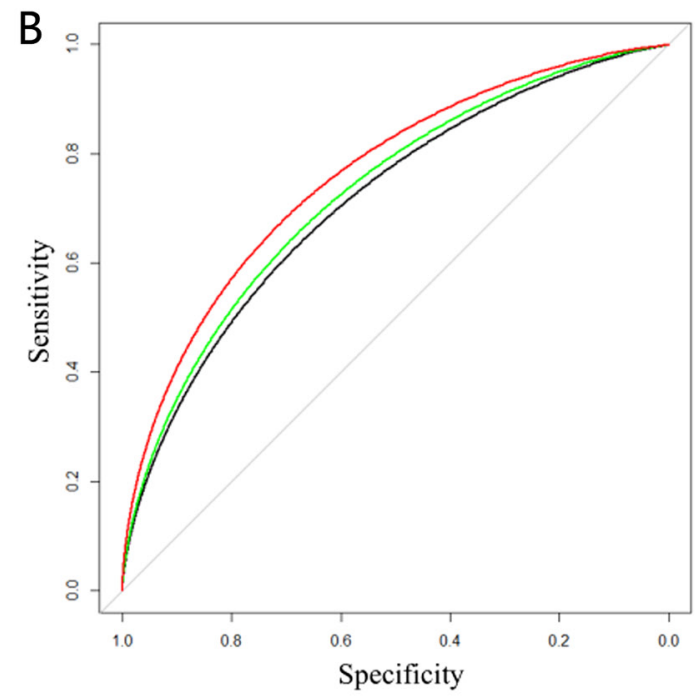

ROC Curve (Area)

Combine (0.724)

- Combine+TG+LDLC (0.738)

Combine+TG+LDLC+Adiponectin (0.779)

\section{Figure 4}

ROC curve of the association of adiponectin with pre-diabetes in cluster (A). Black curve, combined mode (represents the predictive ability of age, $\mathrm{BMI}$, and SBP for pre-diabetes), the AUC is 0.640; green curve, combined mode added with TG and LDL-C, the AUC is 0.665; red curve, combined mode added with TG, LDL-C, and adiponectin, the AUC is 0.682. ROC curve of association of adiponectin with diabetes in cluster 4 (B). Black curve, combined mode (represents the predictive ability of age, BMI, and SBP for diabetes), the AUC is 0.724; green curve, combined mode added with TG and LDL-C, the AUC is 0.738; red curve, combined mode added with TG, LDL-C, and adiponectin, the AUC is 0.779 .
The ROC curve in our study showed that the AUC valve increased after adding TG, LDL-C, and adiponectin to cluster 1 and cluster 4 , which suggested that adiponectin could strengthen the prediction of future pre-diabetes in young-healthy people and future diabetes onset in obese people. A 10-year prospective study including 912 participants concluded that adiponectin was associated with newly onset pre-diabetes and T2DM (28). However, the participants in that study were middle-aged adults in a rural area of Korea, and thus the conclusions might not applicable to people of various ages and races.

Our study has several limitations. First, the newly diagnosis of diabetes were not classified. However, most type 1 diabetes is diagnosed in childhood or during early adulthood. The average age of the participants in our study was $45.7 \pm 14.0$ years old, thus we considered that the newly diagnosed diabetes in our study was mainly T2DM. Second, confounding factors, such as smoking history, alcohol, and education were not excluded. Third, this crosssectional study cannot conclusively determine causality or exclude biases and confounding caused by unmeasured variables. Besides, the precise time-course of adiponectin concentrations in plasma during the development of diabetes remains to be determined. In addition, only Chinese population data was collected in this study, thus the conclusion may not be applied to other ethnic groups.

Collectively, our results showed that an increasing serum adiponectin level was an independent protective factor for pre-diabetes and diabetes in the young-healthy population and for diabetes in the obese population. Adiponectin might play an important role in abnormal glucose metabolism. These effects could be influenced by lipids and other confounding factors, and might not be applicable to the whole population. Our results indicated that we need to pay attention to decreased adiponectin levels in young-healthy and obese people during clinical practice and sub-classification might help to improve patient management.

\section{Declaration of interest}

The authors declare that there is no conflict of interest that could be perceived as prejudicing the impartiality of the research reported.

\section{Funding}

This work was supported by the Guangdong Provincial Scientific and Technological Funds (grant number 2017B20209002); the Guangdong Province Science and Technology Applied Science and Technology Research and Development Special Fund Project (grant number 2020A1515011256). These funding associations had no role in study design, data collection and analysis, decision to publish, or in the preparation of the manuscript. 


\section{Ethical approval}

The experimental procedure described here was approved by the Ethics Committee of the Sun Yat-sen University ([2019] No. 38) and is congruent with the Declaration of Helsinki.

\section{Consent to participate}

Signed informed consent was obtained from every patient who participated.

\section{Author contribution statement}

Xun Gong and Lili You contributed equally to this work and should be regarded as co-first authors. Li Yan and Meng Ren contributed equally to this work as co-corresponding authors.

\section{Acknowledgement}

The authors express sincere appreciation to all participants of the study.

\section{References}

1 Banerjee A, Khemka VK, Roy D, Poddar J, Roy TKS \& Karnam SA. Role of serum adiponectin and vitamin $\mathrm{D}$ in prediabetes and diabetes mellitus. Canadian Journal of Diabetes 201741 259-265. (https://doi. $\operatorname{org} / 10.1016 / j . j c j d .2016 .10 .006)$

2 Li Y, Teng D, Shi X, Qin G, Qin Y, Quan H, Shi B, Sun H, Ba J, Chen B, et al. Prevalence of diabetes recorded in mainland China using 2018 diagnostic criteria from the American Diabetes Association: national cross sectional study. BMJ 2020369 m997. (https://doi.org/10.1136/ bmj.m997)

3 Tabak AG, Herder C, Rathmann W, Brunner EJ \& Kivimaki M. Prediabetes: a high-risk state for diabetes development. Lancet 2012 379 2279-2290. (https://doi.org/10.1016/S0140-6736(12)60283-9)

4 Adiyaman SC, Ozer M, Saydam BO \& Akinci B. The role of adiponectin in maintaining metabolic homeostasis. Current Diabetes Reviews 2020 16 95-103. (https://doi.org/10.2174/1573399815666190702155733)

5 Adiponectin NT. Role in physiology and pathophysiology. International Journal of Preventive Medicine 202011 136. (https://doi. org/10.4103/ijpvm.IJPVM_193_20)

6 Jaganathan R, Ravindran R \& Dhanasekaran S. Emerging role of adipocytokines in Type 2 diabetes as mediators of insulin resistance and cardiovascular disease. Canadian Journal of Diabetes 201842446. e1-456.e1. (https://doi.org/10.1016/j.jcjd.2017.10.040)

7 Weyer C, Funahashi T, Tanaka S, Hotta K, Matsuzawa Y, Pratley RE \& Tataranni PA. Hypoadiponectinemia in obesity and type 2 diabetes: close association with insulin resistance and hyperinsulinemia. Journal of Clinical Endocrinology and Metabolism 200186 1930-1935. (https:// doi.org/10.1210/jcem.86.5.7463)

$8 \mathrm{LiS}$, Shin HJ, Ding EL \& van Dam RM. Adiponectin levels and risk of type 2 diabetes: a systematic review and meta-analysis. JAMA 2009302 179-188. (https://doi.org/10.1001/jama.2009.976)

9 Lai H, Lin N, Xing Z, Weng H \& Zhang H. Association between the level of circulating adiponectin and prediabetes: a meta-analysis. Journal of Diabetes Investigation 20156 416-429. (https://doi. org/10.1111/jdi.12321)

10 Kong SE, Kang YE, Joung KH, Lee JH, Kim HJ \& Ku BJ. Plasma adiponectin levels in elderly patients with prediabetes. Endocrinology and Metabolism 201530 326-333. (https://doi.org/10.3803/ EnM.2015.30.3.326)

11 Shand BI, Scott RS, Lewis JG, Elder PA \& Frampton CM. Comparison of indices of insulin resistance with metabolic syndrome classifications to predict the development of impaired fasting glucose in overweight and obese subjects: a 3-year prospective study. International Journal of Obesity 200933 1274-1279. (https://doi. org/10.1038/ijo.2009.169)

12 Snehalatha C, Mukesh B, Simon M, Viswanathan V, Haffner SM \& Ramachandran A. Plasma adiponectin is an independent predictor of type 2 diabetes in Asian indians. Diabetes Care 200326 3226-3229. (https://doi.org/10.2337/diacare.26.12.3226)

13 Xiong XF, Yang Y, Wei L, Xiao Y, Li L \& Sun L. Identification of two novel subgroups in patients with diabetes mellitus and their association with clinical outcomes: a two-step cluster analysis. Journal of Diabetes Investigation 202112 1346-1358. (https://doi.org/10.1111/ jdi.13494)

14 Anjana RM, Baskar V, Nair ATN, Jebarani S, Siddiqui MK, Pradeepa R, Unnikrishnan R, Palmer C, Pearson E \& Mohan V. Novel subgroups of type 2 diabetes and their association with microvascular outcomes in an Asian Indian population: a data-driven cluster analysis: the INSPIRED study. BMJ Open Diabetes Research and Care 20208 e001506. (https://doi.org/10.1136/bmjdrc-2020-001506)

15 American Diabetes Association. 2. Classification and diagnosis of diabetes: standards of medical care in diabetes-2019. Diabetes Care 2019 42 S13-S28. (https://doi.org/10.2337/dc19-S002)

16 Snijder MB, Heine RJ, Seidell JC, Bouter LM, Stehouwer CD, Nijpels G, Funahashi T, Matsuzawa Y, Shimomura I \& Dekker JM. Associations of adiponectin levels with incident impaired glucose metabolism and type 2 diabetes in older men and women: the hoorn study. Diabetes Care 200629 2498-2503. (https://doi.org/10.2337/ dc06-0952)

17 Christou GA \& Kiortsis DN. Adiponectin and lipoprotein metabolism. Obesity Reviews 201314 939-949. (https://doi.org/10.1111/obr.12064)

18 Li Y, Feng D, Esangbedo IC, Zhao Y, Han L, Zhu Y, Fu J, Li G, Wang D, Wang Y, et al. Insulin resistance, beta-cell function, adipokine profiles and cardiometabolic risk factors among Chinese youth with isolated impaired fasting glucose versus impaired glucose tolerance: the BCAMS study. BMJ Open Diabetes Research and Care 20208 e000724. (https://doi.org/10.1136/bmjdrc-2019-000724)

19 Jiang Y, Owei I, Wan J, Ebenibo S \& Dagogo-Jack S. Adiponectin levels predict prediabetes risk: the pathobiology of prediabetes in a biracial cohort (POP-ABC) study. BMJ Open Diabetes Research and Care 20164 e194. (https://doi.org/10.1136/bmjdrc-2016-000194)

20 Song SO, Han SJ, Kahn SE, Leonetti DL, Fujimoto WY \& Boyko EJ. Leptin and adiponectin concentrations independently predict future accumulation of visceral fat in nondiabetic Japanese Americans. Obesity 202129 233-239. (https://doi.org/10.1002/oby.23035)

21 Liu W, Zhou X, Li Y, Zhang S, Cai X, Zhang R, Gong S, Han X \& Ji L. Serum leptin, resistin, and adiponectin levels in obese and non-obese patients with newly diagnosed type 2 diabetes mellitus: a populationbased study. Medicine 202099 e19052. (https://doi.org/10.1097/ MD.0000000000019052)

22 Banerjee A, Sharma D, Trivedi R \& Singh J. Treatment of insulin resistance in obesity-associated type 2 diabetes mellitus through adiponectin gene therapy. International Journal of Pharmaceutics 2020 583 119357. (https://doi.org/10.1016/j.ijpharm.2020.119357)

23 Mather KJ, Funahashi T, Matsuzawa Y, Edelstein S, Bray GA, Kahn SE, Crandall J, Marcovina S, Goldstein B, Goldberg R, et al. Adiponectin, change in adiponectin, and progression to diabetes in the diabetes prevention program. Diabetes 200857 980-986. (https://doi. org/10.2337/db07-1419)

24 Kizer JR, Arnold AM, Strotmeyer ES, Ives DG, Cushman M, Ding J, Kritchevsky SB, Chaves PH, Hirsch CH \& Newman AB. Change in circulating adiponectin in advanced old age: determinants and impact on physical function and mortality. The Cardiovascular Health Study All Stars Study. Journals of Gerontology: Series A, Biological Sciences and Medical Sciences 201065 1208-1214. (https://doi.org/10.1093/ gerona/glq122) https://ec.bioscientifica.com https://doi.org/10.1530/EC-21-0235
(C) 2021 The authors Published by Bioscientifica Ltd
This work is licensed under a Creative Commons Attribution-NonCommercial-NoDerivatives 4.0 International License.ifica com at $04 / 26 / 2023$ 02:20:22PM 
25 Fagerberg B, Kellis D, Bergstrom G \& Behre CJ. Adiponectin in relation to insulin sensitivity and insulin secretion in the development of type 2 diabetes: a prospective study in 64-year-old women. Journal of Internal Medicine 2011269 636-643. (https://doi.org/10.1111/j.13652796.2010.02336.x)

26 Coimbra S, Brandão Proença J, Santos-Silva A \& Neuparth MJ. Adiponectin, leptin, and chemerin in elderly patients with type 2 diabetes mellitus: a close linkage with obesity and length of the disease. BioMed Research International 20142014 701915. (https://doi. $\operatorname{org} / 10.1155 / 2014 / 701915)$
27 de Vegt F, Dekker JM, Jager A, Hienkens E, Kostense PJ, Stehouwer CD, Nijpels G, Bouter LM \& Heine RJ. Relation of impaired fasting and postload glucose with incident type 2 diabetes in a Dutch population: the Hoorn Study. JAMA 2001285 2109-2113. (https://doi.org/10.1001/ jama.285.16.2109)

28 Cho NH, Ku EJ, Jung KY, Oh TJ, Kwak SH, Moon JH, Park KS, Jang HC, Kim YJ \& Choi SH. Estimated association between cytokines and the progression to diabetes: 10-year follow-up from a community-based cohort. Journal of Clinical Endocrinology and Metabolism 2020105 dgz171. (https://doi.org/10.1210/clinem/dgz171)

Received in final form 15 August 2021

Accepted 6 October 2021

Accepted Manuscript published online 6 October 2021 (c) 2021 The authors Published by Bioscientifica Ltd
This work is licensed under a Creative Commons Attribution-NonCommercial-NoDerivatives 4.0 elnternationad dicense ifica.com at 04/26/2023 02:20:22PM 